\title{
Mesures automatisées de chaleur spécifique à basse température
}

\author{
J. Pinel, C. Lebeau et A. Raboutou \\ I.N.S.A., Dépt. de Génie Physique, 35031 Rennes Cedex, France \\ (Reçu le 9 juillet 1979, révisé le 1er octobre 1979, accepté le 2 octobre 1979)
}

\begin{abstract}
Résumé. - Le système calorimétrique et électronique décrit permet la mesure et le calcul de la chaleur spécifique $C$ en fonction de la température $T$ entre 2 et $30 \mathrm{~K}$. L'ensemble des données obtenues par la méthode de chauffage continu, est traité en temps réel à l'aide d'un micro-ordinateur afin d'obtenir directement la courbe $C(T)$. Le traitement comporte un filtrage numérique qui réduit le bruit des mesures. La technique est illustrée par des résultats sur $\mathrm{Hg}$ et $\mathrm{Pb}$.
\end{abstract}

\begin{abstract}
In this report we describe a calorimetric and electronic system which allows the measurement and the calculus of the specific heat $C$ as a function of the temperature $T$ (between $2 \mathrm{~K}$ and $30 \mathrm{~K}$ ). The data, obtained by the continuous warming method, are processed in real time with the aid of a microcomputer allowing the curve $C(T)$ to be directly obtained. Numeric filtering has been introduced to reduce measurement noise. The method is illustrated by results on $\mathrm{Hg}$ and $\mathrm{Pb}$.
\end{abstract}

1. Introduction. - Lors d'une mesure de capacité calorifique $C$, il est important de pouvoir disposer des résultats pendant l'expérience. C'est pratiquement indispensable lors de la recherche des discontinuités liées aux transitions de phase. Le système de mesure présenté ici remplit cette condition. Il est basé sur un micro-ordinateur, Celui-ci se charge de l'acquisition des données (fournies par le thermomètre) et du traitement de ces données. Il se charge aussi de la gestion des appareils et des périphériques de sortie. Pour donner un exemple pratique, le système est capable de prendre les données toutes les secondes, de leur faire subir un filtrage numérique et de sortir sur table traçante la courbe $C(T)$. Par rapport à un appareillage utilisant un ordinateur plus puissant [1], ce système a l'avantage d'être bien moins coûteux en matériel ou en temps d'immobilisation pour les calculs. De plus, il est totalement autonome et peut être utilisé auprès d'un poste de manipulation, comme un appareil de mesure classique.

Fig. 1. - Calorimètre : 1) passage étanche pour fils de mesure ; 2) tube pour pression d'hélium dans les soufflets ; 3) soufflets servant d'interrupteur thermique ; 4) feuille de cuivre liée thermiquement à l'échantillon; 5) deux barres verticales de soutien; 6) résistances thermométriques ;7) chaufferette ;8) fils de nylon ; 9) joint d'indium ; 10) pièces de fermeture sous vide.

[Calorimeter : 1) glass-metal vacuum seal for measurement leads ; 2) tube for helium pressure in the bellows ; 3 ) bellows for heat switching ; 4) copper foil ; 5) two vertical bars sustaining the assembly ; 6) thermometer ; 7) heater ; 8) nylon threads ; 9) indium 0 ring ; 10) pieces ensuring the vacuum sealing,]
2. Calorimétrie. - Le matériel cryogénique a déjà été décrit [2]. Le calorimètre a la particularité d'être entièrement fermé sous vide (Fig. 1). A basse température, le cryopompage assure un excellent vide d'isolement. On évite ainsi toute intervention de gaz d'échange. Cet appareillage a donné toute satis-

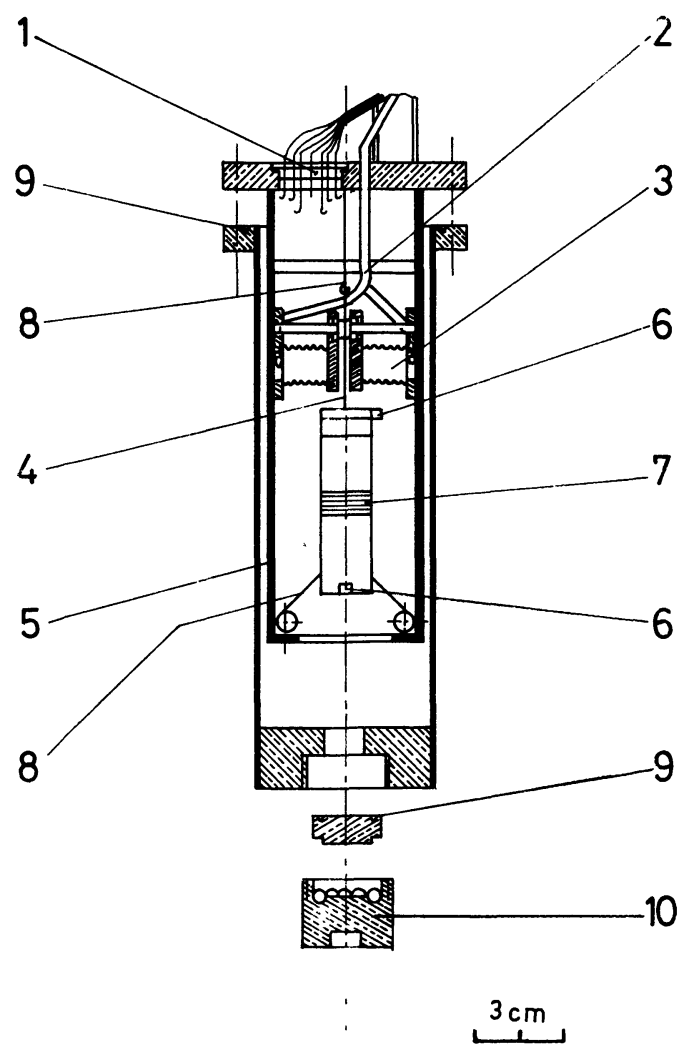


faction entre $2 \mathrm{~K}$ et $30 \mathrm{~K}$. Les dimensions du calorimètre permettent de le placer dans un solénoïde supraconducteur pour des mesures en champ magnétique tout en laissant un volume intérieur suffisant pour loger des échantillons d'environ $10 \mathrm{~cm}^{3}$.

La méthode de chauffage continu consiste à envoyer une puissance thermique $P$ constante dans l'échantillon et à mesurer sa température à intervalles de temps $\tau$ petits et réguliers. On réalise ainsi un échantillonnage de la courbe $T(t)$. La dérivée donne $C(T)=P \tau / m \dot{T}$. La densité de points est typiquement de 1000 par degré pour $\tau=2 \mathrm{~s}$. Grâce à cette grande densité de points, la méthode est particulièrement bien adaptée au traitement des données.

Notons que dans cette méthode, l'échantillon n'a jamais atteint son équilibre thermique. Il existe des écarts entre sa température $T_{\mathrm{E}}$, la température mesurée $T_{\mathrm{M}}$ et celle du support d'échantillon $T_{\mathrm{S}}$; les ordres de grandeur de ces écarts peuvent être analysés à l'aide d'une analogie électrique. Les capacités thermiques de l'échantillon, du support chauffant et du thermomètre ont pour équivalents des capacités $C$. Les résistances thermiques échantillon-support et échantillon-thermomètre ont pour équivalents des résistances électriques $R$. La résistance thermique support-thermomètre est négligée : elle est très grande lorsque les deux éléments sont suffisamment éloignés dans le montage. Supposant, en première approximation, les éléments $R$ et $C$ indépendants de $T$, la réponse à un échelon produit par le générateur de courant $P$, est une rampe (une fois terminée la région transitoire). Il apparaît que $T_{\mathrm{M}}(t)$ est décalée dans le temps par rapport à $T_{\mathrm{E}}(t)$ d'une quantité $R_{\mathrm{T}} C_{\mathrm{T}}$ $\left(R_{\mathrm{T}}\right.$ résistance thermomètre-échantillon, $C_{\mathrm{T}}$ capacité $\mathrm{du}$ thermomètre). Cette quantité faible dans un montage classique du thermomètre conduit à un décalage qu'on peut estimer inférieur à $10^{-3} \mathrm{~K}$. Ceci est confirmé par le repérage de la transition du $\mathrm{Hg}$ à $4,158 \mathrm{~K}$. En début d'expérience, l'écart entre $T_{\mathrm{E}}$ et la réponse idéale (c'est-à-dire pour $T_{\mathrm{E}}=T_{\mathrm{M}}=T_{\mathrm{S}}$ ) est important. L'écart dépend du montage ; il s'évalue expérimentalement en enregistrant la réponse $T_{\mathrm{M}}(t)$ à une impulsion de chauffage. Pour $\mathrm{Hg}$ les transitoires donnent une erreur inacceptable entre $2,8 \mathrm{~K}$ et $3 \mathrm{~K}$ pour une expérience commençant à $2,8 \mathrm{~K}$.

3. Matériel électronique. - Le circuit de mesure comporte un thermomètre constitué d'une résistance carbone parcourue par un courant $I_{\mathrm{T}}$ ajustable $($ de $0,5 \mu \mathrm{A}$ à $50 \mu \mathrm{A})$. La tension a ses bornes $V_{\mathrm{T}}$ est mesurée à l'aide d'un voltmètre numérique (exemple : Hewlett Packard A 3490 ; résolution : $1 \mu \mathrm{V}$; 200000 points). La température est obtenue à partir de $R=V_{\mathrm{T}} / I_{\mathrm{T}}$ et d'une loi d'étalonnage. La sensibilité $\mathrm{du}$ thermomètre est bonne jusqu'à $30 \mathrm{~K}$.

Le circuit de commande comporte une chaufferette (fil de Constantan) parcourue par un courant $I_{\mathrm{CH}}$ programmable. Pour minimiser les fuites thermiques, on n'utilise que 3 fils de liaison. On peut montrer que $P=V_{\mathrm{CH}} I_{\mathrm{CH}}$ avec $V_{\mathrm{CH}}$ la tension mesurée aux bornes de la chaufferette plus un fil.

Un système d'acquisition de données complet a été mis au point (Fig. 2). Il peut être utilisé avec tout appareil de mesure possédant des sorties numériques codées. Le système est basé sur la carte SBC 80/10 INTEL $\left({ }^{1}\right)$ dont l'architecture se fait autour du microprocesseur 8080 et de 3 bus : un bus de données ( 8 bits), un bus d'adresses (16 bits) et un bus de contrôle (6 bits). La carte réunit les éléments essentiels d'un microordinateur : unité centrale (8080), mémoires RAM (2111) et REPROM (2708), entréessorties parallèle (8255) et série (8251) programmables, horloge (18 $432 \mathrm{MHz}$ ), buffers (8226) pour les bus. L'interface série permet de connecter directement un télétype (ce qui permet l'impression et la perforation des résultats). L'interface parallèle est utilisée pour la commande d'un générateur de courant (TAKEDA-RIKEN 6141). La carte possède un logiciel de 78 instructions et un moniteur suffisant pour l'implantation, le test et l'exécution du programme.

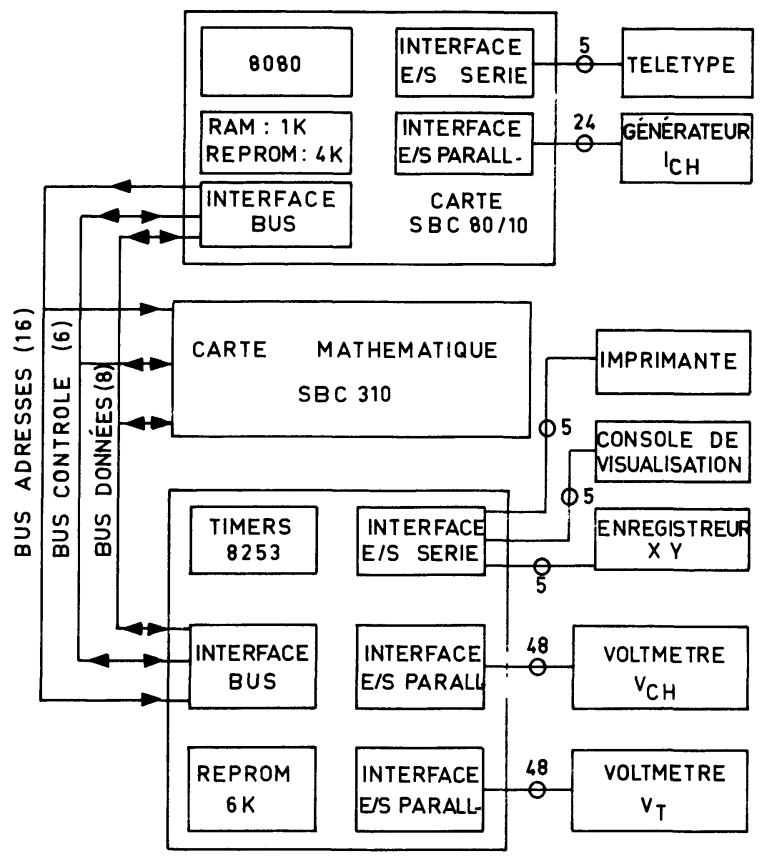

Fig. 2. - Synoptique du système d'acquisition de données.

[Block diagram of the data acquisition system.]

Cette carte a été complétée par, d'une part, une carte mathématique (INTEL SBC 310) et d'autre part, une carte $\left({ }^{2}\right)$ qui contient une extension mémoire (6 kilo-octets de REPROM), une interface bus fournissant les signaux nécessaires au fonctionnement de la carte et des interfaces entrées-sorties. Parmi celles-ci on distingue les interfaces série pour la con-

( $\left.{ }^{1}\right)$ Les fabricants sont donnés à titre indicatif. Il en existe d'autres proposant des produits similaires.

$\left({ }^{2}\right)$ Le détail du câblage est disponible auprès des auteurs. 
nection d'une table traçante numérique (HP $7221 \mathrm{~A}$ ), d'une imprimante rapide (TTY 43) et d'une console de visualisation. Un boîtier (8253) comprenant 3 timers programmables définit les vitesses de transmission convenant à chaque interface et sert aussi de référence de temps pour la mesure. Les interfaces parallèles pour la connection aux voltmètres sont constituées de deux boîtiers $8255(2 \times 24$ bits chacun $)$. Sont utilisés : 4 bits pour le code de l'appareil ; 30 bits pour la lecture des digits en BCD et de la polarité ; 4 bits pour la gamme de mesure ; 1 bit pour le signal fin de mesure ; 1 bit pour la mise en service de l'interface et 1 bit pour le déclenchement des appareils. En définitive, tout appareil ayant $2 \times 10^{7}$ points au maximum peut être connecté. Le code identifiant l'appareil permet de sélectionner, dans les programmes d'acquisition les parties spécifiques à chaque appareil.

4. Logiciel. - Le logiciel (Fig. 3) débute par une initialisation qui se fait de façon conversationnelle rendant facile l'emploi du programme. L'utilisateur est invité à fournir les paramètres : masse de l'échantillon $(m)$, courant dans le thermomètre $\left(I_{\mathrm{T}}\right)$, courant initial dans la chaufferette $\left(I_{\mathrm{CH}}\right)$, intervalle de temps entre deux prises de données $(\tau), 5$ coefficients de la loi d'étalonnage $T(R)$ et, éventuellement, coefficients nécessaires au filtrage numérique. Les échelles pour le tracé de la courbe $C(T)$ sont également mémorisées.

La suite du programme comporte la prise des données, les calculs et la sortie des résultats toutes les $\tau$ secondes. Chaque nouveau cycle de mesure est

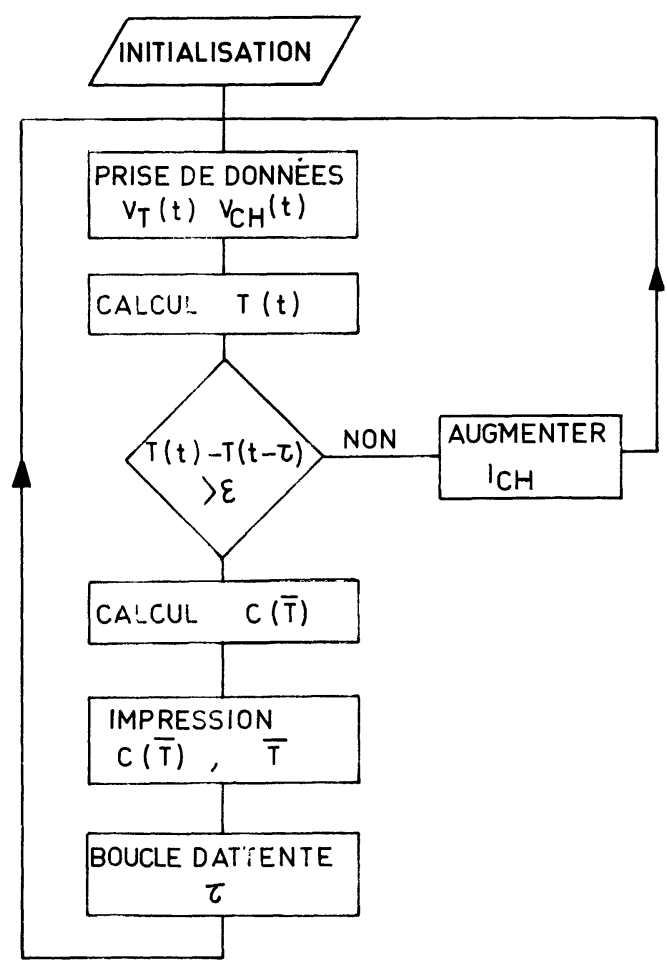

Fig. 3. - Organigramme du programme d'acquisition cyclique.

[Flow-chart of the cyclical acquisition program.] obtenu soit par surveillance d'une horloge (8253) dans une boucle d'attente, soit à la suite d'une interruption provoquée par cette horloge. La valeur de $\tau$ peut être mesurée de façon précise.

A partir des deux données $V_{\mathrm{T}}(t)$ et $V_{\mathrm{CH}}(t)$, où $t$ représente l'instant de la prise de données, les calculs sont effectués en virgule flottante (mantisse de 24 bits, exposant 8 bits). Il faut noter que $V_{\mathrm{CH}}(t)$ varie très peu au cours d'une mesure et qu'on peut la mesurer à des intervalles de temps bien plus grand que $\tau$. Le sous-programme de calcul de $T(t)$ fait appel à la loi d'étalonnage de la résistance carbone (2). Celle-ci contient un calcul de logarithme que nous avons mis au point et testé quant à sa précision et sa rapidité. Le programme teste la vitesse de croissance de $T$ car lorsqu'elle devient trop petite (par exemple inférieure à $2 \mathrm{mK}$ entre 2 points successifs) la dispersion de $C(T)$ augmente fortement et il faut augmenter $I_{\mathrm{CH}}$. Le programme effectue le calcul de $C(\bar{T})=P \tau / m \Delta T$ où $\Delta T=T(t)-T(t-\tau)$ et $2 \bar{T}=T(t)+T(t-\tau)$.

Les deux valeurs $C(\bar{T})$ et $\bar{T}$ sont envoyées sur la table traçante. Le programme d'impression réalise un tracé du cadre et des échelles. Le calcul, le pointé des intervalles sur les axes sont réalisés en fonction des valeurs extrêmes fixées pour le cadre. Le tracé des commentaires peut se faire également, ce qui rend automatique tout le tracé de la courbe (cf. Figs. 4 et 5). Notons que les résultats peuvent également être perforés sur ruban ou stockés sur disque souple pour un traitement en différé. L'ensemble du logiciel $\left({ }^{3}\right)$ occupe $6 \mathrm{~K}$ de REPROM.

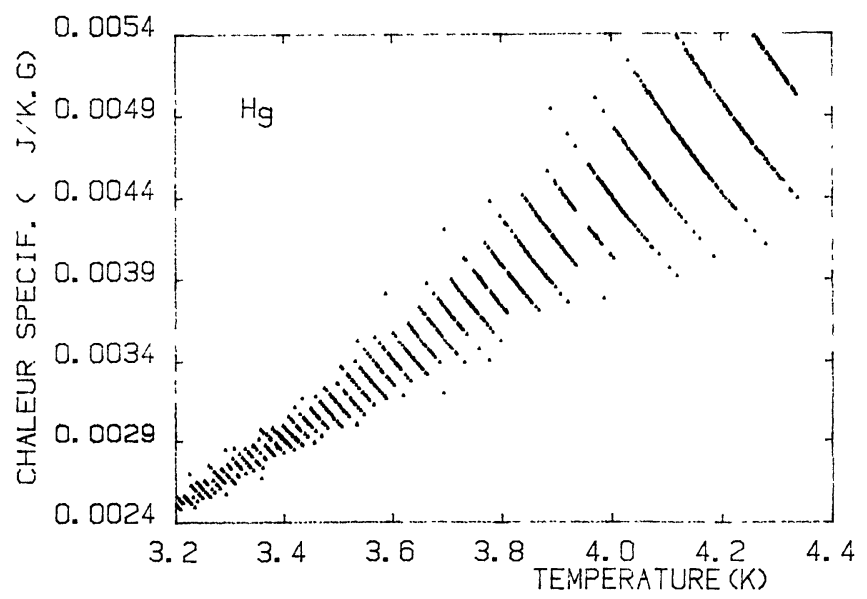

Fig. 4. - Résultats obtenus en temps réel avec $\tau=2 \mathrm{~s}$.

[Results obtained in real time with $\tau=2 \mathrm{~s}$.]

5. Traitement des résultats. - Plusieurs mesures tests sur du $\mathrm{Hg}$ et du $\mathrm{Pb}$ ont été faites en changeant notamment $\tau$. La figure 4 montre la courbe pour le $\mathrm{Hg}$ entre $3,2 \mathrm{~K}$ et $4,4 \mathrm{~K}(m=77,82 \mathrm{~g}, P=144 \mu \mathrm{W}$,

$\left({ }^{3}\right)$ Les programmes peuvent être fournis sur demande. 


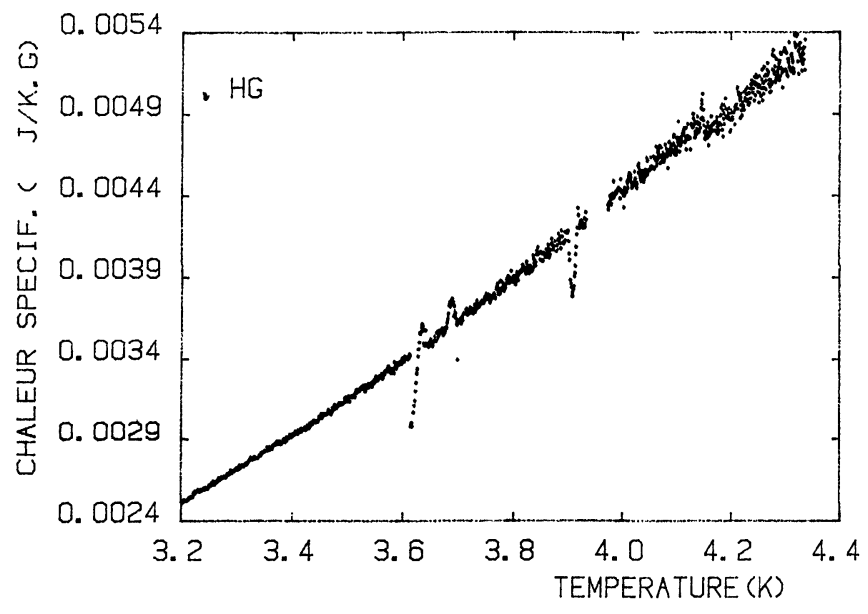

Fig. 5. - Résultats obtenus après filtrage numérique des données identiques à celles de la figure 4 .

[Results obtained after a numeric filtering of the same data as in figure 4.]

$\tau=2 \mathrm{~s})$. Cette courbe résulte de données brutes $V_{\mathrm{T}}(t)$ pour lesquelles la dispersion est particulièrement grande. Par exemple, au niveau de $4 \mathrm{~K}$, les conditions de manipulations sont telles que l'écart entre 2 points successifs est de $10 \mu \mathrm{V}$. Compte tenu que le voltmètre utilisé a une résolution de $1 \mu \mathrm{V}$ cela entraîne la dispersion observée sur $C(T)$ de l'ordre de $20 \%$.

Il est évident sur cette courbe, où en particulier la discontinuité n'apparaît pas, que les résultats ne peuvent être utilisés tels quels. On diminue bien entendu la dispersion des points en optimisant les paramètres de la manipulation notamment par l'emploi d'une sonde de meilleure sensibilité. Cependant, même de telles données brutes peuvent être traitées et exploitées. En traitement différé, on utilise les techniques de lissage habituelles. A ce propos, il faut noter que l'opération dérivation $(\Delta T / \Delta t)$ augmente la dispersion des points. Il est préférable d'opérer le lissage sur les données brutes $T(t)$. Nous avons pu [3] par cette méthode, mettre en évidence de très faibles écarts. Cependant, l'intérêt du microcalculateur est de pouvoir traiter les données en temps réel. Nous avons utilisé un filtre numérique en temps réel défini par [4] :

$$
\begin{array}{r}
T_{\mathrm{F}}(t)=A_{-N^{\prime}} T\left(t-N^{\prime} \tau\right)+\cdots+A_{-1} T(t-\tau)+ \\
+A_{0} T(t)
\end{array}
$$

où $A_{-N^{\prime}}, \ldots, A_{0}$ sont des coefficients de pondération caractéristiques du filtre choisi. Pour le signal $\mathrm{e}^{j \omega t}$, la fonction de transfert en $\omega$ est la fonction périodique $(\Delta \omega=2 \pi / \tau)$ :

$$
F_{-N^{\prime}, 0}(\omega)=\sum_{n=-N^{\prime}}^{0} A_{n} \mathrm{e}^{j n \omega \tau}
$$

Choisissant une fonction de transfert $F(\omega)$, les coeffi- cients $A_{n}$ doivent minimiser la distance séparant $F_{-N^{\prime}, 0}(\omega)$ et $F(\omega)$, alors :

$$
A_{n}=\frac{\tau}{2 \pi} \int_{-\pi / \tau}^{\pi / \tau} F(\omega) \mathrm{e}^{-j n \omega \tau} \mathrm{d} \omega .
$$

En pratique, on oblige la fonction $F(\omega)$ à respecter la condition $(F(\omega))_{\omega=0}=1$ avec laquelle les coefficients deviennent $A_{n}^{*}=A_{n} / \sum_{-N^{\prime}}^{0} A_{i}$.

Plus $\tau$ est petit, donc $\Delta \omega$ élevé, plus la dispersion des résultats sera élevée. Pour éliminer les points les plus dispersés on prend un filtre passe-bas défini par :

$$
\begin{array}{ll}
0 \leqslant \omega \leqslant \omega_{1} & F(\omega)=1 \\
\omega_{1} \leqslant \omega \leqslant \omega & F(\omega)=1+\cos \pi \frac{\omega-\omega_{1}}{\omega_{2}-\omega_{1}} \\
\omega_{2} \leqslant \omega \leqslant \pi / \tau & F(\omega)=0 .
\end{array}
$$

La partie sinusoïdale permet d'éviter toute discontinuité de $F(\omega)$ et facilite son approche par $F_{-N^{\prime}, 0}(\omega)$. Le calcul donne :

$$
A_{n}=\frac{\pi}{2 n} \frac{\sin n \omega_{1} \tau+\sin n \omega_{2} \tau}{\pi^{2}-n^{2} \tau^{2}\left(\omega_{2}-\omega_{1}\right)^{2}}
$$

Nous avons fait quelques tests de filtre pour différents $\omega_{1}$ et $\omega_{2}$, le filtre étant d'autant plus sévère que $\omega_{1}$ et $\omega_{2}$ sont pris petits devant $\Delta \omega$ (avec $\tau=2 \mathrm{~s}$, $\left.\Delta \omega=3,14 \mathrm{~s}^{-1}\right)$.

Dans le logiciel, on inclut un sous-programme qui pondère $\operatorname{par} A_{0}^{*} \ldots A_{-N^{\prime}}^{*}$, les valeurs de

$$
T(t) \ldots T\left(t-N^{\prime} \tau\right)
$$

puis en déduit la valeur filtrée $T_{\mathrm{F}}(t)$. La courbe 5 montre le résultat obtenu sur le $\mathrm{Hg}$ avec un filtre tel que $\omega_{1}=0,1 \mathrm{~s}^{-1}, \omega_{2}=0,15 \mathrm{~s}^{-1}, N^{\prime}=20$. Les résultats correspondent aux mêmes données brutes $V_{\mathrm{T}}(t)$ que sur la figure 4. L'amélioration apportée par le filtre permet notamment de faire apparaître la discontinuité de $C(T)$ au niveau de $4,16 \mathrm{~K}(\sim 2 \%$ théoriquement). Les accidents aux niveaux de $3,7 \mathrm{~K}$ et $3,9 \mathrm{~K}$ correspondent au manque de continuité dans le relevé des points (changement de puissance).

Des mesures ont aussi été faites sur $\mathrm{Pb}$ placé dans un champ magnétique $H_{\mathrm{a}}$. Pour ces mesures, nous nous sommes attachés à montrer comment on pouvait localiser les discontinuités à l'aide d'un filtrage numérique en temps réel. La valeur absolue de $C$ peut présenter des incertitudes liées à des incertitudes sur $P$ et sur l'étalonnage de la sonde. Sans filtre numérique, les courbes présentant beaucoup de dispersion et sont difficilement exploitables. Un filtre numérique met en évidence les discontinuités (Fig. 6) qui apparaissent aux transitions de phases lorsque $H_{\mathrm{a}}=H_{\mathrm{c}}\left(T_{\mathrm{c}}\right)$. Pour $H_{\mathrm{a}}=0$, la discontinuité est de l'ordre de $5 \%$. Ces résultats obtenus pour des champs de 49, 157, 200 et 300 Oe sont en accord 


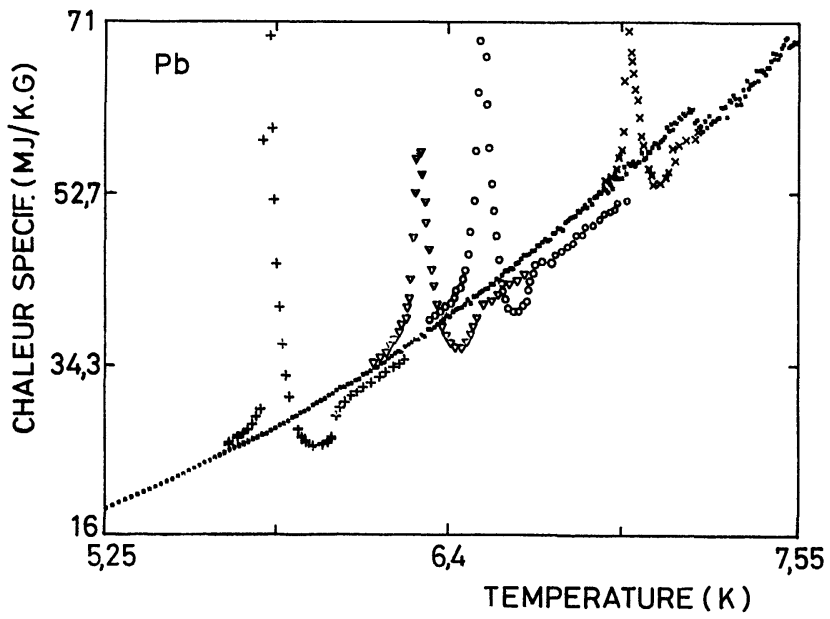

Fig. 6. - Résultats obtenus en temps réel avec filtrage numérique des données. Les champs appliqués sont en œrsteds : $0(\square)$, $49(\times), 157(0), 200(\nabla), 300(+)$.

[Results obtained in real time with numeric filtering of the data. The applied fields are in œrsteds : $0(\square), 49(\times), 157(0), 200(\nabla)$ and $300(+)$.]

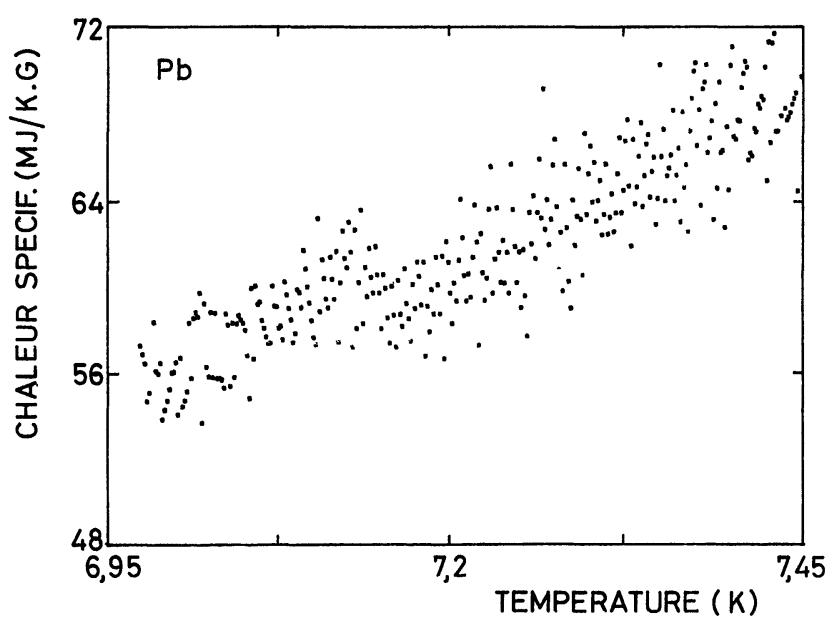

Fig. 7. - Résultats obtenus en temps réel avec filtrage numérique des données, $\tau=3 \mathrm{~s}$ et $P=182 \mu \mathrm{W}$.

[Results obtained in real time with the numeric filtering of the data, $\tau=3 \mathrm{~s}$ and $P=182 \mu \mathrm{W}$.]

avec ceux obtenus par ailleurs [5]. Ces courbes ont été obtenues pour

$m=48,91 \mathrm{~g}, \quad I_{\mathrm{T}}=10 \mu \mathrm{A}, \quad P=726 \mu \mathrm{W}, \quad \tau=3 \mathrm{~s}$

et le filtre numérique $\omega_{1}=0,1 \mathrm{~s}^{-1} ; \omega_{2}=0,15 \mathrm{~s}^{-1}$; $N^{\prime}=20$. Il faut noter que la qualité de la courbe $C(T)$ dépend des caractéristiques du filtre (c'est-à-dire

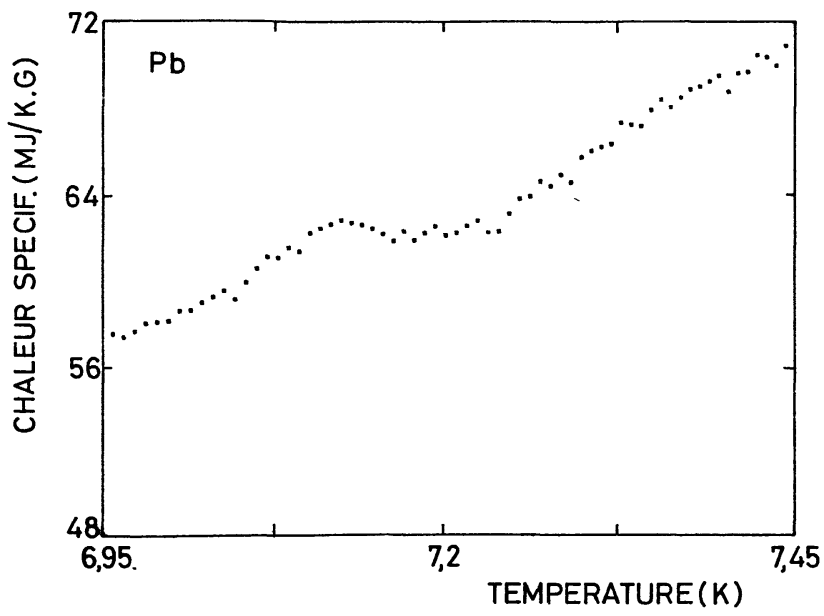

Fig. 8. - Résultats obtenus en temps réel avec le même filtre que celui de la figure 7 et avec $\tau=4$ s et $P=786 \mu \mathrm{W}$.

[Results obtained in real time with the same filter as in figure 7 and with $\tau=4 \mathrm{~s}$ and $P=786 \mu \mathrm{W}$.]

de $\omega_{1}, \omega_{2}$ et $N^{\prime}$ ), et du choix de $P$ et $\tau$. Pour un filtre donné, augmenter $\tau$ ou $P$ revient à diminuer la dispersion sur $C$. Ceci est équivalent à diminuer $\Delta \omega$, c'est-à-dire le nombre de composantes ayant des fréquences élevées dans le développement en série de Fourier de $F(\omega)$. Le filtrage en est d'autant facilité. Ainsi, les résultats de la figure 7 correspondent au même filtre que ci-dessus (Fig. 6) avec $\tau=3 \mathrm{~s}$, mais $P=182 \mu \mathrm{W}$. Un filtre plus sévère apparaît comme nécessaire. Par contre, le même filtre apparaît comme convenable dans la figure 8 avec $P=786 \mu \mathrm{W}$ et $\tau=4 \mathrm{~s}$.

D'autres types de filtres peuvent aussi être utilisés. Des filtres plus efficaces [4] et adaptés à un cas donné peuvent être implantés en acceptant une complication du logiciel. Dans la pratique, il s'avère que le filtre proposé est suffisant dans la plupart des cas notamment en ce qui concerne l'obtention des premiers résultats.

6. Conclusion. - Nous avons décrit un dispositif automatisé de mesure de chaleur spécifique. Celui-ci a nécessité la mise au point d'un système d'acquisitions de données à l'aide d'un microprocesseur. Les avantages principaux du système sont sa mobilité et son faible coût. Le traitement des données en temps réel permet d'obtenir un premier tracé de $C(T)$. Ceci allège énormément les mesures thermiques à basse température qui sont normalement longues à mettre en œuvre.

\section{Bibliographie}

[1] Moses, D., Ben-Aroya, O. et Lupu, N., Rev. Sci. Instrum. 48 8 (1977) 1098.

[2] Pinel, J. et Lebeau, C., J. Phys. E 5 (1972) 688.

[3] Lebeau, C. et Pinel, J., Phys. Status Solidi (a) 23 (1974) 453.
[4] RADIX, J. C., Introduction au filtrage numérique (Eyrolles Paris) 1970

[5] Chanin, G. et Torre, J. P., Phys. Rev. B 5 (1972) 4357. 